\title{
Number and density of percolating clusters $\dagger$
}

\author{
C M Newman $\neq \mid$ and L S Schulman§ \\ \$ Mathematics Department, University of Arizona, Tucson, AZ 85721, USA \\ $\S$ Physics Department, Technion, Haifa, Israel
}

Received 7 November 1980, in final form 17 February 1981

\begin{abstract}
Properties of infinite clusters in general percolation models are investigated. The number of infinite clusters is either zero, one or infinity. Results are found on the density of the various kinds of infinite clusters. In particular, 'filamentary' and 'rough' clusters, which individually do not have non-zero density, are studied with an eye toward ascertaining whether these exist in some models, and hence whether an infinity of infinite clusters is possible. Conjectures are presented on the behaviour of systems with an infinity of infinite clusters under scale transformations, and also on the way in which the presence of such clusters influences the prevalence of large finite clusters.
\end{abstract}

\section{Introduction}

It has generally been believed that with the onset of percolation, only a single infinite cluster exists. Heuristic arguments in favour of this assertion have been presented (Kikuchi 1970) although some authors in discussing large but finite volumes have distinguished between 'having some cluster span the volume' and 'having a finite fraction of the sites part of the same cluster'. In two dimensions it is known that, at most, a single infinite cluster exists (Harris 1960, Fisher 1961).

In this article we present rigorous results on the number and density of infinite clusters and on other properties as well. We show that, under quite general circumstances, the only possibilities for the number of infinite clusters are: zero, one, infinity. Specifically, with probability one, it is impossible to have two or any greater finite number of distinct infinite clusters.

Besides settling the question of having a finite number of infinite clusters our result raises the intriguing possibility that there can occur an infinity of infinite clusters. In pursuit of this issue we present a number of properties necessarily possessed by such clusters, should they exist. It must be confessed that our initial goal in studying these properties was to find a contradiction, but having failed to do so, we now suspect that an infinity of infinite clusters can occur, perhaps in sufficiently high dimension, perhaps with anisotropic connection probabilities.

Detailed proofs of some of our results are being presented elsewhere (Newman and Schulman 1981) and in this article we only outline the precise arguments. In addition, we give material of a heuristic nature which does not appear in the other article.

† Supported by United States-Israel Binational Science Foundation Grant 1573.

$\|$ Alfred P Sloan Fellow, on leave from Indiana University. Supported in part by NSF Grant MCS77-206783. I Address for 1980/1981: IBM Thomas J Watson Research Center, Yorktown Heights, NY 10598, USA. 


\section{Rigorous results on infinite clusters}

Although we phrase most of our statements in terms of the site percolation problem on a $d$-dimensional square lattice, our results are more general and apply to any system satisfying the following three hypotheses:

(a) Translation invariance: If $P$ is the probability distribution for configurations of the lattice (a measure on the sample space $\Omega=\{0,1\}^{\mathbf{z}^{d}}$ ) and if $T_{j}$ is translation in any lattice direction $j \in \mathbf{Z}^{d}$, then $T_{j} P=P$.

(b) Translation ergodicity. If $W$ is a translationally invariant event, then it either always occurs or never occurs (up to sets of measure zero). That is, if for $j \neq 0$, and $W \subset \Omega, T_{j} W=W$ then either $P(W)=1$ or $P(W)=0$.

(c) Anything can happen locally. If $W$ is an event for which $P(W) \neq 0$, and if $W^{\prime}$ is an event whose configurations differ from those of $W$ only in some particular finite region, then $P\left(W^{\prime}\right) \neq 0$.

Hypothesis (b) is especially powerful for our purposes. It is the same principle that plays a role in establishing that, although for finite systems with a given site occupation probability $p$ there may sometimes exist a cluster spanning the entire volume and sometimes not, as the volume goes to infinity the existence or nonexistence of such a cluster becomes a sure property as a function of $p$ alone. This is because the event 'having an infinite cluster' is a translationally invariant set.

Hypothesis (b) has as a consequence the following property which is more closely related to the usual concept of ergodicity. Let $X$ be a random variable, $X: \Omega \rightarrow \mathbf{R}$, and let $R_{n} \subset \mathbf{Z}^{d}$ be the cube of edge length $n$ centred on the origin. Finally, let $E(X)$ be the expectation of $X$, i.e. $E(X)=\int_{\Omega} X(\omega) \mathrm{d} P(\omega)$. Then

$$
\lim _{n \rightarrow \infty} n^{-d} \sum_{j \in R_{n}} T_{j} X=E(X) \quad \text { wpo (with probability one). }
$$

For $d=1$ this is the familiar notion of equality of time average and phase space average.

In the site percolation problem, sites on $\mathbf{Z}^{d}$ are independently occupied with probability $p$ and a site $i$ is connected to $j$ if both $i$ and $j$ are occupied and there is a path of nearest-neighbour occupied sites from $i$ to $j$. This system satisfies all three above hypotheses, in particular it satisfies hypothesis (c) because change of status from occupied to unoccupied or vice versa on $n$ sites can at most introduce a factor $[p /(1-p)]^{ \pm n}$ which is non-zero for $0<p<1$. Hypothesis $(b)$ is known to be valid for independent percolation; see Billingsley (1965), Theorem 1.2.

Another system satisfying all three hypotheses is the Ising model with 'spin up' taken to mean occupied. For finite volumes the probability of a given configuration is proportional to

$$
\mathrm{e}^{-\beta H} \quad \text { with } \quad H=-\sum_{i, k} J(j-k) \sigma_{j} \sigma_{k}-h \sum_{j} \sigma_{j}
$$

where $J(l)$ decreases sufficiently rapidly that

$$
\sum_{l}|J(l)|<\infty .
$$

Hypothesis (c) is then satisfied because changes in system configuration in a finite region lead to only finite changes in energy.

Let $C(j)$ be the set of sites connected to $j \in \mathbf{Z}^{d} . C(j)$ will be known as a cluster. For a set $F$ let $|F|$ be the number of sites belonging to $F$. Let $H$ be the set of all clusters. Let 
$H_{0}$ denote the set of infinite clusters, that is

$$
H_{0}=\{C \in H|| C \mid=\infty\} \text {. }
$$

Let $\left|H_{0}\right|$ be denoted $N_{0} ; N_{0}$ is the number of distinct infinite clusters. Our principal result is:

Theorem 1. Either $N_{0}=0$ wpo or $N_{0}=1$ wpo or $N_{0}=\infty$ wpo.

Proof. Let $S(k)$ be the set of all lattice configurations which have exactly $k$ infinite clusters, i.e.,

$$
S(k)=\left\{\omega \in \Omega \mid N_{0}(\omega)=k\right\} .
$$

Suppose for some $k, 1<k<\infty$, the measure of $S(k)$ is non-zero; that is, suppose there is a non-zero probability of finding exactly $k$ infinite clusters. The property of having a particular number of infinite clusters is a translationally invariant property so that by hypothesis (b) it must always (i.e., with probability one) be the case that there are exactly $k$ clusters (parameters such as $p, d$ or $h$ of our examples above are held fixed). Moreover, it can never (i.e., with probability zero) happen that there is exactly one cluster. We prove the theorem by using hypothesis (c) to show a contradiction, namely, that there is positive measure for $S(1)$ if there is for $S(k)$.

Let $R_{n}$ be a $d$-cube of side $n$ centred on the origin. Let $W_{n}$ be the set of lattice configurations (i.e., points of $\Omega$ ) for which there are exactly $k$ infinite clusters and all of them enter $R_{n}$. For sufficiently large $n$ the set $W_{n}$ must have non-zero measure (this follows since $\lim _{n \rightarrow \infty} W_{n}=S(k)$ and if $W_{n}$ had measure zero for all $n$, so would $S(k)$ ). Fixing this $n$, let $W$ be the set of lattice configurations obtained by taking each lattice configuration of $W_{n}$ and forcing all sites inside $R_{n}$ to be occupied (or all spins up for the Ising model) while leaving the configuration unchanged outside $R_{n}$. By hypothesis (c) the measure of $W$ is also non-zero since it differs from $W_{n}$ in only a finite region. But configurations in $W$ have but one cluster, providing the desired contradiction.

Note that the proof breaks down for $k=\infty$ since no $R_{n}$ can have a bit of each cluster.

Let $\rho$ be the fraction of all sites which are part of some infinite cluster. By ergodicity this equals the probability that any particular site (for example, the origin) is part of an infinite cluster.

Proposition. If $N_{0} \neq 0$ then $\rho>0$ (wpo).

Proof. If $\rho=0$ then the probability of any site being part of an infinite cluster is exactly zero, hence the probability of any countable union of sites being part of an infinite cluster is zero. But $\mathbf{Z}^{d}$ is such a countable union. One consequence of this proposition is that if, as is generally believed, $\rho=0$ at $p_{\mathfrak{c}}$ (=percolation threshold) then there is no infinite cluster at $p_{\mathrm{c}}$. (This does not contradict the possibility that the largest cluster in a region of size $n^{d}$ goes to infinity with $n$.)

In studying the possibility of having an infinity of infinite clusters it should be realised that hypotheses (a), (b) and (c) (which are all we use in our proofs) allow for a great variety of site occupation rules in addition to the examples we have given. For this reason it is plausible that some strange clusters could emerge. In particular we have found it useful to distinguish three sorts of infinite cluster, depending on their density. To define the density of a cluster $C$ one wishes to study the limit of $\left|C \cap R_{n}\right| / n^{d}$ for $n \rightarrow \infty$ (recall $\left|R_{n}\right|=n^{d}$ ). Infinite clusters for which the limit superior and limit inferior of this object do not agree, do not have a density defined and we call these rough. When 
the two limits agree and are positive, an infinite cluster is called dense. If the limits agree and are zero, then an infinite cluster is called flamentary. For any set $S$ for which the limit $\left|S \cap R_{n}\right| / n^{d}$ exists, we call that limit the density of the set.

By arguments similar to those used in proving theorem 1, we can show:

Theorem 2. The number of filamentary clusters is either zero or $\infty$. The number of rough clusters is either zero or $\infty$. The number of dense clusters is either zero or one. If there are any rough clusters, there is no dense cluster. The union of all filamentary clusters has a well defined density which is positive if there are any filamentary clusters. The union of all rough clusters has a well defined density which is positive if there are any rough clusters.

We shall not indicate how the entire proof goes, but to show the recurrent use of ergodicity we give part of the proof. Suppose $N_{0}=\infty$ and consider the set of all clusters for which $\lim \inf \left(\left|R_{n} \cap C\right| / n^{d}\right)$ is positive. Part of the proof consists of establishing that there is at most one cluster in this set (for rough clusters this lim inf must be zero). Suppose there were more. Now the upper bound of the set of lim inf's is some number, call it $\bar{Y}$. By ergodicity this number takes the same value for all (up to sets of measure zero) configurations. It is easy to see that there will always (wpo) be pairs of clusters the sum of whose lim inf's exceeds $\bar{Y}$. But then (as in the proof of theorem 1 ) there are lattice configurations (with non-zero measure) for which in some particular $R_{n}$ these clusters merge and have lim inf greater than $\bar{Y}$, a contradiction.

Another interesting property of $N_{0}=\infty$ systems is the existence of 'close encounter' points and 'cutting' points. A close encounter site is one which is empty but if filled would unite two or more previously distinct infinite clusters. A cutting site is an occupied site that is part of an infinite cluster and which if vacated would cause that cluster to break into two or more infinite clusters. Not only do these sites exist but they exist with finite density (by the same argument used in showing that $\rho=0$ implies there is no infinite cluster).

Another property of interest in percolation theory is the surface area of clusters and in particular the surface to volume ratio. We consider two kinds of surface: external surface and total surface. The total surface of a cluster consists of all vacant sites which are at a distance 1 from the cluster. The external surface consists of sites of the total surface which are part of an infinite cluster of vacant sites (where cluster formation for vacant sites obeys the same rules as for occupied sites).

For external surface we have a result which may be considered surprising: if there is any external surface at all, then it has positive density. This implies that the surface to volume ratio is positive and in some sense the surface of the infinite cluster is of the same dimension as the cluster itself. The proof of this assertion involves the same measure theoretic argument used to show that $\rho=0$ implies no infinite cluster.

For total surface we show that the surface to volume ratio is $(1-p) / p$ for independent percolation. This same ratio obtains whether one looks at the total volume of the union of all infinite clusters and the associated surface or whether one looks at the single dense cluster (if there is one). Moreover, when $N_{0}=\infty$ the ratio of close encounter points to cutting points is this same number.

This ratio had been anticipated by Hankey (1978) and Stoll and Domb (1978). Klein and Shamir (1980) had already obtained this result rigorously before us (it was also known to Aizenmann et al (1980)) and their proof also carries over to individual filamentary or rough clusters. The virtue of our proof is the use of a simple duality argument. To apply this we first note that the value $(1-p) / p$ for the surface to volume 
ratio is equivalent to the validity of

$$
E(X Y)=E(X) E(Y)
$$

where $X$ is the indicator function of the event that the origin be occupied and $Y$ is the indicator function of the event that the origin belongs either to the infinite cluster or to its surface. The duality argument is then used to show that $X$ and $Y$ are actually independent random variables. In the case of correlated percolation, where $F K G$ inequalities are valid, we find that $E(X Y) \geqslant E(X) E(Y)$, which implies that the surface to volume ratio is not greater than the ratio of vacant (spin-down) to occupied (spin-up) sites. This is true both for the aggregate of all infinite clusters and for the single dense cluster, if there is one.

Another property that has attracted attention in percolation theory is the behaviour of large blocks of sites and the way in which properties of such blocks fluctuate about the mean. Properties of this sort are of interest for renormalisation group studies of percolation. It turns out that for systems obeying the $F K G$ inequalities (which includes our two examples given above) if a certain correlation function is integrable then the number of sites in $R_{n}$ belonging to infinite clusters has an asymptotically Gaussian distribution as $n \rightarrow \infty$; a similar result applies to the single cluster (if there is one). More precisely let $Z_{n}$ be the deviation of the number of sites in $R_{n}$ belonging to an infinite cluster from its expected value, $\rho n^{d}$. Let $K(i)$ be the correlation function or covariance, $K(i)=$ Prob (both 0 and $i$ belong to infinite clusters) $-\rho^{2}$, and finally suppose

$$
\nu=\sum_{i \in \mathbf{Z}^{d}} K(i)<\infty .
$$

Then the random variable

$$
Z_{n} / n^{d / 2} \nu^{1 / 2}
$$

converges (in distribution) as $n \rightarrow \infty$ to a Gaussian random variable of mean zero and covariance one.

In some sense one expects filamentary or rough clusters to be of lower, perhaps fractal, dimension. To state this more precisely, we have found it useful to define the following quantities:

$$
\begin{aligned}
& G(j)=\operatorname{Prob}(j \text { is connected to } 0) \\
& Q_{n}=n^{-d} \sum_{j \in R_{n}} G(j) .
\end{aligned}
$$

The following properties can be established:

If

$$
\liminf _{n \rightarrow \infty} \sum_{j \in \partial R_{n}} G(j)=0
$$

where $\partial R_{n}$ denotes the surface of $R_{n}$, then $N_{0}=0$. This is because the condition forces $C(0)$, the cluster attached to the origin, to be finite (wpo) which shows that $N_{0}=0$ (as in the proof that $\rho=0$ implies $N_{0}=0$ ).

If

$$
\sum_{j \in \mathbf{Z}^{d}}\|j\|^{-d+1} G(j)<\infty
$$

(with $\|j\|$ being the Euclidean length of $j$ ) then all clusters are filamentary. If $\lim \inf _{n \rightarrow \infty} Q_{n}=0$, then there is no dense cluster. If all clusters are filamentary, then 
$\lim _{n \rightarrow \infty} Q_{n}=0$. Finally, if there is any dense cluster (of density $\rho_{d}>0$ ), then

$$
\lim _{n \rightarrow \infty} Q_{n}=\left(\rho_{d}\right)^{2}>0 \text {. }
$$

Now use $G(j)$ to define a 'mean effective dimension,'

$$
\delta=\sup \left\{\delta^{\prime} \mid \lim _{n \rightarrow \infty} n^{-\delta^{\prime}} \sum_{j \in R_{n}} G(j)=\infty\right\}
$$

and define for each infinite cluster $C$, its effective dimension as,

$$
\underline{\delta}(C)=\sup \left\{\delta^{\prime}\left|\lim _{n \rightarrow \infty} n^{-\delta^{\prime}}\right| C \cap R_{n} \mid=\infty\right\} .
$$

Then it can be shown that for any infinite cluster

$$
\underline{\delta}(C) \leqslant \delta \text { (wpo). }
$$

\section{Scale transformations and renormalisation}

We now speculate on the way in which filamentary clusters would appear under a renormalisation group treatment. Suppose coordinate space scale transformations have been defined for the independent site percolation problem which includes as a parameter the original site occupation probability $p$. Under scale transformations $p=0$ and $p=1$ are expected to be attractors. In the usual situation $p_{c}$, the percolation threshold, is a repeller (on the $p$ axis) but now we must take into account the possibility that (in sufficiently high dimension) there is more than one threshold. For example, it seems plausible that there is some range $p_{\mathrm{c}}^{\prime}<p<p_{\mathrm{c}}^{\prime \prime}$ within which only filamentary clusters are present. The $p_{c}^{\prime}$ would be the threshold for filamentary clusters while $p_{c}^{\prime \prime}$ would mark the onset of a dense cluster (we neglect the possibility of rough clusters in this discussion).

If this were the case, then by the usual arguments, scale transformations starting from $p>p_{c}^{\prime \prime}$ should lead to $p=1$ while those for $p<p_{c}^{\prime}$ should send $p$ to zero. Thus each of these points is a repeller insofar as transformations outside of $\left(p_{c}^{\prime}, p_{c}^{\prime \prime}\right)$ are concerned. But within that range it is not possible for both points to repel, unless there is yet another fixed point, which we assume not to be the case.

Which way does the arrow go-from $p_{c}^{\prime}$ to $p_{c}^{\prime \prime}$ or vice versa? It seems that this depends on the nature of the scale transformation, and in particular we expect that various scale transformations find one or the other kind of critical point invisible. There is nothing startling in this suggestion. For example, a decimation transformation eliminating every second site on an Ising antiferromagnetic would preclude seeing staggered magnetisation.

Our earlier remarks on dimension suggest that filamentary clusters are 'thinner' than the usually contemplated sort and that scale transformations which would hope to capture the order parameter associated with these clusters would need very generous conventions on when two sites are to be considered connected in the scaled system. Such a transformation beginning slightly above $p_{\mathrm{c}}^{\prime}$ and for which $p_{\mathrm{c}}^{\prime}$ is a repeller would go right past $p_{c}^{\prime \prime}$ as if it were not there. Similarly, a convention for site connection which had just the right sensitivity to pick out the onset of dense clusters would find filamentary clusters invisible and if the iteration began from $p$ slightly less than $p_{c}^{\prime \prime}$ it would head towards $p=0$ with no notice of $p_{\mathrm{c}}^{\prime}$. 
This situation highlights one of the problems that invariably confronts the would be renormaliser. For percolation there is ambiguity in the rule to be used in deciding whether a rescaled site is occupied. Our suggestion is that when two conventions give different answers it may not always be appropriate to discard the calculation.

Another possibility is that every point in the interval $\left[p_{c}^{\prime}, p_{c}^{\prime \prime}\right]$ is a fixed point. This situation could arise if there is an infinite correlation length throughout the interval.

\section{Systems which may show filamentarity}

It is rigorously known that two-dimensional site percolation has only dense clusters. It seems reasonable to suppose that with increasing dimension it will be possible for distinct clusters to avoid meeting each other and remain filamentary. A degree of anisotropy in connection rules can also aid in keeping clusters apart. For example, one candidate is directed percolation in three dimensions. With directed bonds, one of these dimensions can be considered a time axis and there is some preliminary numerical evidence (Gerola et al 1980) that filamentarity occurs.

On the other hand, for random graphs (Erdos 1973), which is in some sense an infinite dimensional mean field model, above the percolation threshold there is but one cluster. (This is a system of $N$ points, each connected to any one of the others with probability $x / N$. The value $x=1$ is the critical value for percolation.)

There is an indication that filamentarity may occur when $\beta\left(\rho \sim\left(p-p_{c}\right)^{\beta}\right)$ is unity, which is the case for $d \geqslant 6$. The heuristic argument for this is based on the total volume occupied by a cluster and its surface.

\section{Cluster sizes}

The prevalence of large finite clusters above $p_{\mathrm{c}}$ differs markedly from that below $p_{\mathrm{c}}$ and, of course, from the situation at $p_{\mathrm{c}}$ itself. Let $P_{k}(p)$ be the probability of finding a cluster of exactly $k$ sites (i.e., the probability that $|C(0)|=k$ ). For $p<p_{\mathrm{c}}$,

$$
\log P_{k}=\mathrm{O}(k)
$$

thus $P_{k} \sim \exp (-c k)$ for some $c$. Above $p_{c}$ there is a simple argument (Aizenmann et al 1981) showing that

$$
P_{k}(p) \geqslant \exp \left(-c k^{(d-1) / d}\right)
$$

for some $c$ (not necessarily the same as that above). The argument uses the fact that there is an infinite cluster. Namely, by changes on the surface of a cube of side $k^{1 / d}$ (which 'costs' the probability factor given above) one can separate a large cluster of volume $k$ from the infinite cluster. For $p$ sufficiently large it is also known that there is an upper bound for $P_{k}(p)$ with the same $k$ dependence.

Now a dense cluster should intersect the walls of $R_{n}$ on the order of $n^{d-1}$ places so that it is not surprising that the above estimate is the best possible. However, the ratio of surface intersections of $R_{n}$ to volume intersections for filamentary clusters should be smaller and one could use this too as a definition of dimension (although we do not know how this relates to the other definitions of dimension). Calling this dimension $\delta^{\prime}$ we expect

$$
P_{k}(p) \sim \exp \left(-c k^{\left(\delta^{\prime}-1\right) / \delta^{\prime}}\right)
$$


for $p_{c}^{\prime}<p<p_{c}^{\prime \prime}$. Intuition based on our earlier resuits on cutting points and close encounter points also suggests that when filamentary clusters are present, one is more likely to find big clusters which are not infinite. In some sense the range $p_{\mathrm{c}}^{\prime}<p<p_{\mathrm{c}}^{\prime \prime}$ is partway between criticality and the onset of dense clusters. At criticality there are no infinite clusters but many very large ones. For slightly larger $p$ there are some rather thin infinite clusters and still quite a few very large finite ones.

\section{Remarks on essential singularities}

The above estimates of $P_{k}(p)$ were first found by Kunz and Souillard (1978) and used by them to show the appearance of an essential singularity for a certain (analogue of the partition) function. Recently, Baker and Kim (1980) have found analogous estimates on the growth rate of the coefficients in the expansion of the magnetisation as a function of $h$ (external field) in the Ising model. These estimates provide strong evidence for the existence of a singularity at $h=0$ (hence at a first-order phase transition). It was noted by Baker and Kim and also by Lowe and Wallace (1980) that the growth rate is the same as that expected from the droplet model for first-order phase transitions. In this model one continues the free energy to the metastable range and finds an imaginary part for the free energy. The growth rate imposed by this imaginary part is the same as that seen by Baker and Kim.

We wish to make a technical comment concerning limitations on the use of growth estimates for establishing the possibility and nature of an analytic continuation beyond a singularity even when those estimates are what have provided the proof of the existence of that singularity. It should be noted that this potential analytic continuation is of considerable interest and in the case of Baker and Kim would say a good deal about metastability. The point we wish to make is that changes in Taylor coefficients which are invisible from the point of view of growth estimates may give rise to a natural boundary which stops any analytic continuation.

For the problem studied by Baker and Kim one wishes to know whether for a series

$$
\sum_{k=0}^{\infty} a_{k} z^{k}
$$

with $z=\exp (-2 \beta h)$, analytic continuation is possible around $z=1(h=0)$. The numerical results of Baker and Kim together with droplet model calculations suggest that

$$
a_{k} \sim k^{-g} \exp \left(-c k^{\sigma}\right)
$$

with $g \geqslant 0, c>0,0<\sigma<1$ which is similar to the percolation model growth rate for $P_{k}(p)$ if we take $\sigma=(d-1) / d$. Suppose we define $a_{k}^{\prime}$ by

$$
a_{k}^{\prime}= \begin{cases}\exp \left(-k^{\sigma^{\prime}}\right) & \text { if } k=n ! \text { for some } n \\ 0 & \text { otherwise }\end{cases}
$$

with $\sigma<\sigma^{\prime}<1$; then the series

$$
\sum_{k=0}^{\infty} a_{k}^{\prime} z^{k}
$$

has $|z|=1$ as a natural boundary (this follows from a theorem of Hadamard (Dienes 
1931)). Thus, even if the original series with coefficients $a_{k}$ has an analytic continuation around $z=1$, the new series

$$
\sum_{k=0}^{\infty}\left(a_{k}+a_{k}^{\prime}\right) z^{k}
$$

would have no such analytic continuation. This new series represents an 'invisible' perturbation of the original one since

$$
\left(a_{n}+a_{n}^{\prime}\right) / a_{n}-1 \sim \exp \left[-\mathrm{O}\left(k^{\sigma^{\prime}-\sigma}\right)\right]
$$

\section{References}

Aizenmann M, Delyon F and Souillard B 1981 J. Stat. Phys. to appear 1980 private communication

Baker G A and Kim D 1980 J. Phys. A: Math. Gen. 13 L103

Billingsley P 1965 Ergodic Theory and Information (New York: Wiley)

Dienes P 1931 The Taylor Series (London: OUP)

Erdos P 1973 The Art of Counting. Selected Writings (Cambridge, Mass.: MIT)

Fisher M E 1961 J. Math. Phys. 2620

Gerola H, Schulman L S and Seiden P E 1980 private communication

Harris T E 1960 Proc. Camb. Phil. Soc. 5913

Hankey A 1978 J. Phys. A: Math. Gen. 11 L49

Kikuchi R 1970 J. Chem. Phys. 532713

Kunz H and Souillard B 1978 J. Stat. Phys. 1977

Klein T and Shamir E 1980 Rigorous Results on Infinite Cluster Surfaces in Percolation preprint

Lowe M J and Wallace D J 1980 J. Phys. A: Math. Gen. 13 L381

Newman C M and Schulman L S $1981 J$. Stat. Phys. to appear

Stoll E and Domb C 1978 J. Phys. A: Math. Gen. 11 L57 